CERN-PH-TH/2004-102

\title{
Heating up the cold bounce
}

\author{
Massimo Giovannini \\ Department of Physics, Theory Division, CERN 1211 Geneva 23, Switzerland
}

\begin{abstract}
Self-dual string cosmological models provide an effective example of bouncing solutions where a phase of accelerated contraction smoothly evolves into an epoch of decelerated Friedmann-Robertson-Walker expansion dominated by the dilaton. While the transition to the expanding regime occurs at sub-Planckian curvature scales, the Universe emerging after the bounce is cold, with sharply growing gauge coupling. However, since massless gauge bosons (as well as other massless fields) are super-adiabatically amplified, the energy density of the maximally amplified modes re-entering the horizon after the bounce can efficiently heat the Universe. As a consequence the gauge coupling reaches a constant value, which can still be perturbative.
\end{abstract}




\section{Formulation of the problem}

One of the inspiring symmetries of the pre-big bang scenario is the scale factor duality (SFD), allowing the connection of different cosmological solutions of the low-energy string effective action [1]. For instance, in the string frame description, accelerated solutions with growing curvature are connected via SFD to decelerated Friedmann-Robertson-Walker (FRW) models with decreasing curvature and decreasing dilaton energy density. The same solutions can also be described in the so-called Einstein frame, where the dilaton is not directly coupled to the Einstein-Hilbert term [2]. In this case a solution exhibiting accelerated contraction evolves into a decelerated FRW expansion.

The connection among different solutions provided by the combined action of SFD and time-reversal does not imply an analytical connection of the duality-related solutions, as clearly noted in the early days of the pre-big bang cosmology [3]. In spite of this, it is known that solutions connecting smoothly two duality-related regimes exist in the presence of dilaton potentials which are explicitly invariant under the SFD symmetry $[3,4,5]$. Recently its was shown $[6,7]$ that these models can be derived from a generally covariant action supplemented by a non-local dilaton potential. In the absence of fluid sources and in the Einstein frame metric, these solutions smoothly interpolate between a phase of accelerated contraction valid for negative values of the conformal-time coordinate and a phase of decelerated expansion valid for positive values of the conformal time coordinate. The intermediate high-curvature phase is regular and can be interpreted as cold bounce (CB) since the Universe is never dominated by radiation, be it before or after the bounce. Furthermore, the nature of CB solutions also implies that the dilaton field never becomes constant for $\eta \rightarrow+\infty$ but is always growing logarithmically.

A possible way out is to consider the effects of the high-frequency (small-scale) modes amplified during the epoch preceding the bounce. In the low-energy effective action also Abelian gauge bosons may be present and since their coupling to the dilaton field breaks conformal invariance [8] the various modes of the "photon" field can be super-adiabatically amplified. While inside the horizon the quantum mechanical fluctuations related to these modes are adiabatically damped. On the contrary, as the modes get outside the horizon, their energy density is super-adiabatically amplified, thanks to the coupling of the kinetic term of the gauge bosons to the dilaton field. When the fluctuations re-enter the horizon, after the bounce, their energy density red-shifts as radiation while the energy density of the background, still dominated by the dilaton field, red-shifts as stiff matter. Since the energy density of the background decreases faster than radiation, there will be a moment where the energy density of the radiation background will become dominant, providing a mechanism for the gravitational heating of the cold bounce [9]. Well after the bounce the 
dilaton potential is negligible with respect to the dilaton kinetic term; this means that when radiation starts dominating the dilaton field will be driven to a constant asymptotic value for $\eta \rightarrow+\infty$.

The idea that back-reaction effects can produce dynamically a radiation-dominated background has been previously discussed within different approaches, but not specifically in connection with the problem of dilaton stabilization. The idea of a possible gravitational reheating of the Universe was pointed out by Parker [10, 11] and also, in a related perspective, by Grishchuk [12]. Lukash and Starobinsky [13] considered similar effects but paid particular attention to the process of isotropization of anisotropic solutions by particle production. In more recent times, Ford [14] discussed the problem of production of slightly non-conformally coupled fields in the case of inflationary Universes. Even more recently Peebles and Vilenkin [15] used the mechanism of gravitational reheating in the case of quintessential inflation (see also [16]).

In the following the different steps outlined in the previous paragraphs looked into. In Section 2 the considerations leading to cold bounces are discussed both in the String and in the Einstein frame. In Section 3 the amplification of Abelian gauge bosons are accurately computed. Section 4 describes the gravitational reheating of the cold bounces. The concluding remarks are presented in Section 5. The Appendices contain useful technical results supporting and complementing the results of Sections 2 and 4.

\section{Cold bounces in the string and Einstein frames}

The string frame action leading to cold-bounce solutions $[6,7]$ can be written as

$$
S=-\frac{1}{2 \lambda_{s}^{2}} \int d^{4} x \sqrt{-G} e^{-\varphi}\left[R+G^{\alpha \beta} \partial_{\alpha} \varphi \partial_{\beta} \varphi+V(\bar{\varphi})\right]+S_{\mathrm{m}}
$$

where $\lambda_{s}$ is the string length scale and the metric signature is mostly minus ${ }^{1}$. In Eq. (2.1) $S_{\mathrm{m}}$ account for the possible contribution of matter sources.

The potential $V(\bar{\varphi}(x)$ ), a local function of $\bar{\varphi}$, is instead a non-local function (yet a scalar under general coordinate transformations) of the dilaton owing to the definitions:

$$
V=V\left(e^{-\bar{\varphi}}\right), \quad e^{-\bar{\varphi}(x)}=\int \frac{d^{4} y}{\lambda_{s}^{2}} \sqrt{-G(y)} e^{-\varphi(y)} \sqrt{G^{\mu \nu} \partial_{\mu} \varphi(y) \partial_{\nu} \varphi(y)} \delta(\varphi(x)-\varphi(y)) .
$$

From the metric $G_{\mu \nu}$, the induced metric

$$
\gamma_{\mu \nu}=G_{\mu \nu}-\frac{\partial_{\mu} \varphi \partial_{\nu} \varphi}{(\partial \varphi)^{2}}
$$

\footnotetext{
${ }^{1}$ Greek indices run over the four space-time dimensions, while Latin indices take values over the threedimensional spatial geometry.
} 
and the induced Laplacian

$$
\hat{\nabla}^{2} \varphi=\gamma_{\mu \nu} \nabla^{\mu} \nabla^{\nu} \varphi
$$

can be easily defined. Thus, in terms of Eqs. (2.3) and (2.4), the variation of the action (2.1) with respect to $G_{\mu \nu}$ and $\varphi$ leads, respectively, to the following two equations:

$$
\begin{gathered}
R_{\mu}^{\nu}-\frac{1}{2} \delta_{\mu}^{\nu}+\nabla_{\mu} \nabla^{\nu} \varphi+\frac{1}{2} \delta_{\mu}^{\nu}\left[G^{\alpha \beta} \nabla_{\alpha} \varphi \nabla_{\beta} \varphi-2 G^{\alpha \beta} \nabla_{\alpha} \nabla_{\beta} \varphi-V\right]-\frac{1}{2} e^{-\varphi} \sqrt{(\partial \varphi)^{2}} \gamma_{\mu}^{\nu} I_{1}=\lambda_{s}^{2} e^{\varphi} T_{\mu}^{\nu}, \\
R+2 G^{\alpha \beta} \nabla_{\alpha} \nabla_{\beta} \varphi-G^{\alpha \beta} \partial_{\alpha} \varphi \partial_{\beta} \varphi+V-\frac{\partial V}{\partial \bar{\varphi}}+e^{-\varphi} \frac{\hat{\nabla}^{2} \varphi}{\sqrt{(\partial \varphi)^{2}}} I_{1}-e^{-\varphi} V^{\prime} I_{2}=0,
\end{gathered}
$$

With standard notation, $T_{\mu}^{\nu}$ is the energy-momentum tensor of the sources, while the integrals

$$
\begin{aligned}
& I_{1}=\frac{1}{\lambda_{s}^{3}} \int d^{4} y \sqrt{-G(y)} V^{\prime}\left(e^{-\bar{\varphi}(y)}\right) \delta(\varphi(x)-\varphi(y)), \\
& I_{2}=\frac{1}{\lambda_{s}^{3}} \int d^{4} y \sqrt{-G(y)} \sqrt{\partial_{\mu} \varphi(y) \partial^{\mu} \varphi(y)} \delta^{\prime}(\varphi(x)-\varphi(y)),
\end{aligned}
$$

arise directly from the standard variational procedure.

For a homogeneous, isotropic and spatially flat background,

$$
g_{00}=1, \quad g_{i j}=-a_{s}^{2}(t) \delta_{i j}, \quad \varphi=\varphi(t), \quad T_{0}^{0}=\rho_{s}(t), \quad T_{i}^{j}=-p_{s}(t) \delta_{i}^{j},
$$

the evolution equations (2.5) and (2.6) greatly simplify and become perfectly local in time. In particular, from Eq. (2.2)

$$
e^{-\bar{\varphi}}=e^{-\varphi} a_{s}^{3},
$$

having absorbed into $\varphi$ the dimensionless constant $-\ln \left(\int d^{3} y / \lambda_{s}^{3}\right)$, associated with the (finite) comoving spatial volume. In the class of backgrounds specified by Eq. (2.8), the time and space components of Eq. (2.5), and the dilaton equation (2.6), lead to the following set of equations

$$
\begin{aligned}
& \dot{\bar{\varphi}}^{2}-3 H_{s}^{2}-V=e^{\bar{\varphi}} \bar{\rho}_{s}, \\
& \dot{H}_{s}=\dot{\varphi} H_{s}+\frac{1}{2} e^{\bar{\varphi}} \bar{p}_{s}, \\
& 2 \ddot{\bar{\varphi}}-\dot{\bar{\varphi}}^{2}-3 H_{s}^{2}+V-\frac{\partial V}{\partial \bar{\varphi}}=0 \\
& \dot{\bar{\rho}}_{s}+3 H \bar{p}_{s}=0
\end{aligned}
$$

where the dot denotes derivation with respect to the cosmic time in the string frame and where $H_{s}=\dot{a}_{s} / a_{s}$. In Eqs. (2.10)-(2.13) the following quantities have been defined:

$$
\bar{\rho}_{s}=a_{s}^{3} \rho_{s}, \quad \bar{p}_{s}=a_{s}^{3} p_{s} .
$$


In the string frame (and in the absence of fluid sources) the system of Eqs. (2.10)-(2.12) admits various cold-bounce solutions and some examples are provided in the Appendix (see also [7]). An interesting case is given, for instance, by the following solution:

$$
\begin{aligned}
& V(\bar{\varphi})=-V_{0} e^{4 \bar{\varphi}}, \\
& a_{s}(t)=a_{0}\left[\tau+\sqrt{\tau^{2}+1}\right]^{1 / \sqrt{3}}, \\
& \bar{\varphi}=\varphi-3 \ln a_{s}(t)=-\frac{1}{2} \log \left(1+\tau^{2}\right)+\varphi_{0},
\end{aligned}
$$

where

$$
\tau=\frac{t}{t_{0}}, \quad t_{0}=\frac{e^{-2 \varphi_{0}}}{\sqrt{V_{0}}} .
$$

The value of $t_{0}^{-1}$ sets the maximal value of the Hubble barameter, $H_{s}(t)$, at the bounce. The value of $\varphi_{0}$ sets the typical value of the dilaton at the bounce.

Using Eq. (2.16), it can be checked that $a_{s}(t)=a_{s}^{-1}(-t)$ as implied by the invariance of the solution under scale factor duality. Other duality-invariant solutions of the system of Eqs. (2.10)-(2.13) are reported in Appendix together with a more extensive discussion of the cold-bounce solution.

The conformal time coordinate is the same in both Einstein and string frames [2]. Thus, in order to swiftly perform the correct transition from string to Einstein frame, it is appropriate to rewrite the system of Eqs. (2.10)-(2.13) in the conformal time coordinate, i.e.

$$
a_{s}\left(\eta_{s}\right) d \eta_{s}=d t_{s}
$$

After this coordinate change, Eqs. (2.10)-(2.13) can be written as:

$$
\begin{aligned}
& \varphi^{\prime 2}+6 \mathcal{H}_{s}^{2}-6 \mathcal{H}_{s} \varphi^{\prime}=V a_{s}^{2}+a^{2} e^{\varphi} \rho_{s}, \\
& \mathcal{H}_{s}^{\prime}=\mathcal{H}_{s} \varphi^{\prime}-2 \mathcal{H}_{s}^{2}+\frac{e^{\varphi / 2}}{2} a_{s}^{2} p_{s}, \\
& 2 \varphi^{\prime \prime}+4 \mathcal{H}_{s} \varphi^{\prime}-6 \mathcal{H}_{s}^{\prime}-6 \mathcal{H}_{s}^{2}+V-\frac{\partial V}{\partial \varphi}=0, \\
& \rho_{s}^{\prime}+3 \mathcal{H}_{s}\left(\rho_{s}+p_{s}\right)=0
\end{aligned}
$$

where $\mathcal{H}_{s}=a_{s}^{\prime} / a_{s}$ and the prime denotes derivation with respect to $\eta_{s}$. As already mentioned, the dilaton and the conformal time coordinate do not change in the transition from Einstein to string frames

$$
\eta_{e}=\eta_{s}=\eta, \quad \varphi_{e}=\varphi_{s}=\varphi
$$

where $\eta$ and $\varphi$ denote the common values of the conformal time coordinate and of the dilaton in both frames. The scale factor, the Hubble parameter, and the energy and pressure 
densities do change in the transition from string to Einstein frame:

$$
a_{s}=e^{\varphi / 2} a, \quad \mathcal{H}_{s}=\mathcal{H}+\frac{\varphi^{\prime}}{2}, \quad \rho_{s}=e^{-2 \varphi} \rho, \quad p_{s}=e^{-2 \varphi} p,
$$

where $a, \mathcal{H}, \rho$ and $p$ are the Einstein frame quantities while $\varphi$ and $\eta$ will be, respectively, the common values of the dilaton and of the conformal time coordinate in both frames.

Applying the transformations given in (2.25), Eqs. (2.20) and (2.23) lead to the Hamiltonian constraint and to the conservation equation:

$$
\begin{aligned}
& 6 \mathcal{H}^{2}=\rho a^{2}+\frac{1}{2} \varphi^{\prime 2}+e^{\varphi} V a^{2} . \\
& \rho^{\prime}+3 \mathcal{H}(\rho+p)-\frac{\varphi^{\prime}}{2}(\rho-3 p)=0 .
\end{aligned}
$$

Equations (2.21) and (2.22) lead, respectively, to

$$
\begin{aligned}
& \varphi^{\prime \prime}+2 \mathcal{H} \varphi^{\prime}+2 \mathcal{H}^{\prime}+4 \mathcal{H}^{2}=p a^{2}, \\
& \varphi^{\prime \prime}+2 \mathcal{H} \varphi^{\prime}+\frac{\partial V}{\partial \varphi}-V+6 \mathcal{H}^{\prime}+6 \mathcal{H}^{2}-\frac{\varphi^{\prime 2}}{2}=0 .
\end{aligned}
$$

By linear combination of Eqs. (2.28) and (2.29), the two remaining equations of the system can be obtained, namely:

$$
\begin{aligned}
& 4 \mathcal{H}^{\prime}+2 \mathcal{H}^{2}=-p a^{2}-\left(\frac{\varphi^{\prime 2}}{2}-e^{\varphi} V a^{2}\right)-e^{\varphi} \frac{\partial V}{\partial \bar{\varphi}} a^{2}, \\
& \varphi^{\prime \prime}+2 \mathcal{H} \varphi^{\prime}+\frac{1}{2}(\rho-3 p) a^{2}+e^{\varphi}\left(V a^{2}-\frac{1}{2} \frac{\partial V}{\partial \bar{\varphi}} a^{2}\right)=0 .
\end{aligned}
$$

Equations (2.26)-(2.27), and (2.30)-(2.31) form a closed set of equations whose solutions will now be analysed first in the absence of fluid sources.

The Einstein frame equations can also be obtained, in generic $d$ spatial dimensions, by transforming the string frame action into the Einstein frame and by doing the functional variation directly in the Einstein frame variables [7]. Clearly the two approaches lead to the same result expressed in eqs. (2.26)-(2.27) and (2.30)-(2.31).

In the Einstein frame, the fluid evolution and the dilaton are directly coupled in the conservation equation (2.13). In the particular case of radiation, since the energy-momentum tensor is traceless, the coupling to the dilaton disappears from Eq. (2.13).

The solution presented in Eqs. (2.15)-(2.17) cannot be written in simple analytical terms in the Einstein frame, but Eqs. (2.26) and (2.27) can be integrated numerically. In the Einstein frame description, the asymptotics of the solution (2.15)-(2.17) are

$$
a(\eta) \simeq a_{-} \sqrt{-\frac{\eta}{2 \eta_{0}}}, \quad a_{-}=e^{-\varphi_{0} / 2} \sqrt{\frac{2(\sqrt{3}+1)}{\sqrt{3}}},
$$




$$
\begin{aligned}
& \varphi_{-}=\varphi_{0}-\ln 2-\sqrt{3} \ln \left(\frac{\sqrt{3}+1}{\sqrt{3}}\right)-\sqrt{3} \ln \left(-\frac{\eta}{2 \eta_{0}}\right), \\
& \mathcal{H}_{-}=\frac{1}{2 \eta}, \quad \varphi_{-}^{\prime}=-\frac{\sqrt{3}}{\eta},
\end{aligned}
$$

for $\eta \rightarrow-\infty$, and

$$
\begin{aligned}
& a(\eta) \simeq a_{+} \sqrt{\frac{\eta}{2 \eta_{0}}}, \quad a_{+}=e^{\varphi_{0} / 2} \sqrt{\frac{2(\sqrt{3}-1)}{\sqrt{3}}} \\
& \varphi_{+}=\varphi_{0}-\ln 2-\sqrt{3} \ln \left(\frac{\sqrt{3}-1}{\sqrt{3}}\right)+\sqrt{3} \ln \left(\frac{\eta}{2 \eta_{0}}\right), \\
& \mathcal{H}_{+}=\frac{1}{2 \eta}, \quad \varphi_{+}^{\prime}=\frac{\sqrt{3}}{\eta},
\end{aligned}
$$

for $\eta \rightarrow+\infty$. The branch of the solution denoted by minus describes an accelerated contraction, since the first derivative of the scale factor is negative while the second is positive. The branch of the solution denoted with plus describes, in the Einstein frame, a decelerated expansion, since the first derivative of the scale factor is positive while the derivative is negative. In both branches the dilaton grows and its derivative is always positive-definite (i.e. $\varphi_{ \pm}^{\prime}>0$ ) as required by the present approach to bouncing solutions.

Recalling that, in the Einstein frame, the potential term of Eq. (2.15) and its derivative with respect to $\bar{\varphi}$ are

$$
\frac{\partial V}{\partial \bar{\varphi}} e^{\varphi} a^{2}=4 V e^{\varphi} a^{2}, \quad V=-V_{0} \frac{e^{-2 \varphi}}{a^{12}},
$$

Eqs. (2.26)-(2.31) can be numerically integrated across the bounce.

By setting initial conditions on the asymptotic solution, the integration of the non-linear problem can be performed by checking that the constraint of Eq. (2.26) is always accurately satisfied. For instance, in the case of the numerical solutions illustrated in Fig. 2.1, the constraint is satisfied with a precision of $10^{-8}$ over the whole range of the solution.

In the light of the subsequent applications, it is useful to parametrize the numerical evolution of the solution in terms of the gauge coupling and of its derivatives

$$
g(\eta)=e^{\varphi / 2}, \quad \varphi^{\prime}(\eta)=2 \frac{g^{\prime}}{g} .
$$

In order to find cold-bounce solutions, the system of Eqs. (2.26)-(2.31) is integrated in the absence of sources. The initial conditions are chosen in such a way that the gauge coupling and the curvature scale, in Planck units, are always minute. Defining $\eta_{i}$ as the initial time of integration

$$
g_{i}=g\left(\eta_{i}\right) \ll 1, \quad \frac{\mathcal{H}\left(\eta_{i}\right)}{M_{\mathrm{P}}}=\frac{\mathcal{H}_{i}}{M_{\mathrm{P}}} \ll 1 .
$$


The Hamiltonian constraint of Eq. (2.26) is enforced on the initial data and the initial conditions for the scale factor coincide with the solution (2.32). One important parameter of the solutions is the width of the bounce, which will be conventionally denoted by $\eta_{0}$. The specific value of $\eta_{0}$ for a given solution can be determined numerically. Typical values of $\eta_{0}$ range between $\eta_{\mathrm{P}}$ and $100 \eta_{\mathrm{P}}$. Furthermore, it turns out that, numerically:

$$
\mathcal{H}_{\max } \simeq \frac{0.6}{\eta_{0}}, \quad \varphi_{\max }^{\prime}=\frac{3.8}{\eta_{0}} .
$$

These expessions are rather accurately verified for all the sets of initial conditions analysed in the present investigation. In the following numerical examples the initial time of integration will always be denoted by $\eta_{\mathrm{i}}$. For each integration the initial value of the gauge coupling, $g_{i}$ will also be specified.

In Fig. 2.1 the results of the numerical integration are illustrated, for different sets of initial conditions in terms of the evolution of the dilaton (i.e. twice the Neperian logarithm ${ }^{2}$ of the gauge coupling) and of the scale factor. In Fig. 2.2 the evolution of the derivative of the dilaton and of the Hubble parameter is reported in conformal time.

In the left plot of Fig. 2.1, the numerical solution for the evolution of the scale factor is compared with the asymptotic solutions provided by the analytical expressions derived in Eqs. (2.32) and (2.33). The analytical approximation reproduces rather well with the numerical result. The reason for this agreement stems from the right plot in Fig. 2.2 where the logarithm (in ten basis) of the potential terms is compared with the logarithm of the dilaton kinetic energy. Far from the bouncing region the potential is always orders of magnitude smaller than the dilaton kinetic energy. Hence the asymptotic solutions are, in practice, a good approximation to the full solution not only for $\eta \rightarrow \pm \infty$ but also, in less restrictive terms, away from the bounce. The solutions of Fig. 2.1 and of the following plots will be presented in terms of the ratio $\eta / \eta_{\mathrm{P}}$ where $\eta_{\mathrm{P}}=\sqrt{2} / M_{\mathrm{P}}$. In natural Planck units, $16 \pi G=2 / M_{\mathrm{P}}^{2}=1$ and $\eta_{\mathrm{P}}=1$.

The solutions illustrated so far have realistic features. The most notable feature being the possibility of describing the transition between the pre-big bang and the post-big bang at relatively small curvature. However, various difficulties still have to be faced. The first problem is that the Universe emerging after the bounce is cold. The solution, even if expanding, is not dominated by radiation as one would like it to be at some later stage of the life of the Universe. The second problem is connected with the evolution of the gauge coupling. In order to trust our perturbative extimates, it should happen that $g(\varphi)<1$ throughout all the stages of the model with an aymptotically (constant) value of the order

\footnotetext{
${ }^{2}$ In the present paper the logarithm in ten basis will be denoted by log while the Neperian logarithm will be denoted by $\ln$.
} 


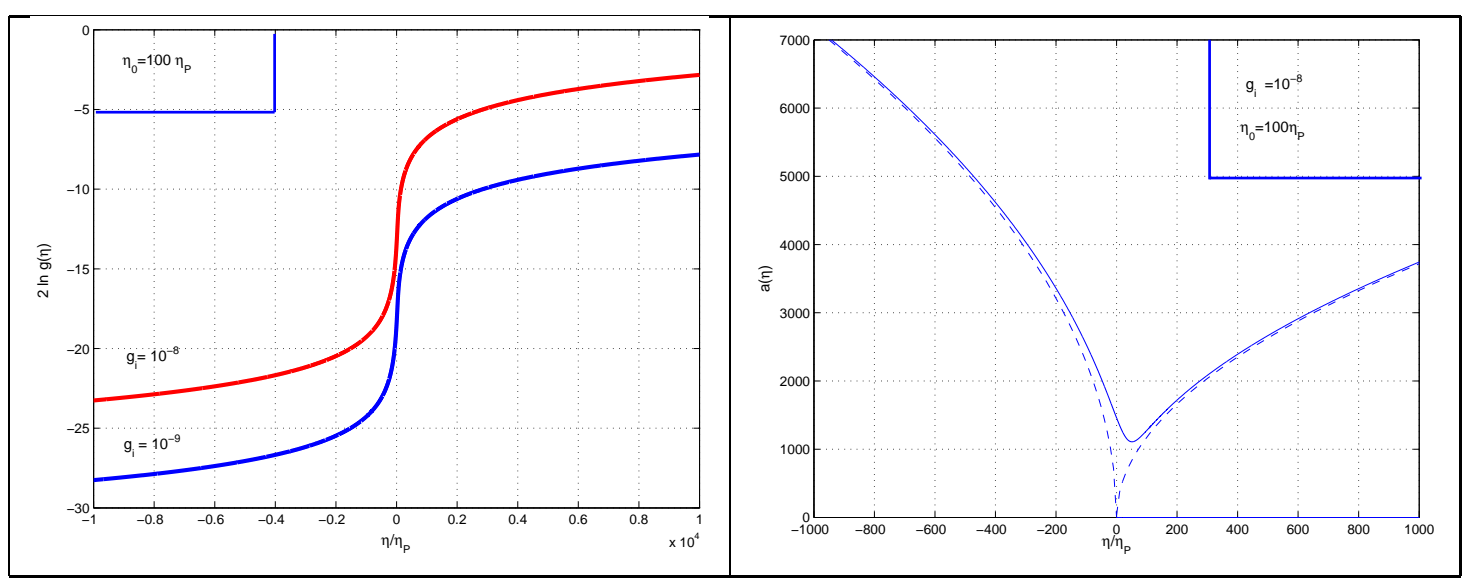

Figure 2.1: In the left plot the result of the integration is illustrated in terms of the (Neperian) logarithm of the gauge coupling. In the right plot the scale factor obtained numerically (solid line) is compared with the analytical (asymptotic) solutions (dashed lines) following from Eqs. (2.32) and (2.33). For both integrations $\eta_{\mathrm{i}}=-10^{7} \eta_{\mathrm{P}}$.
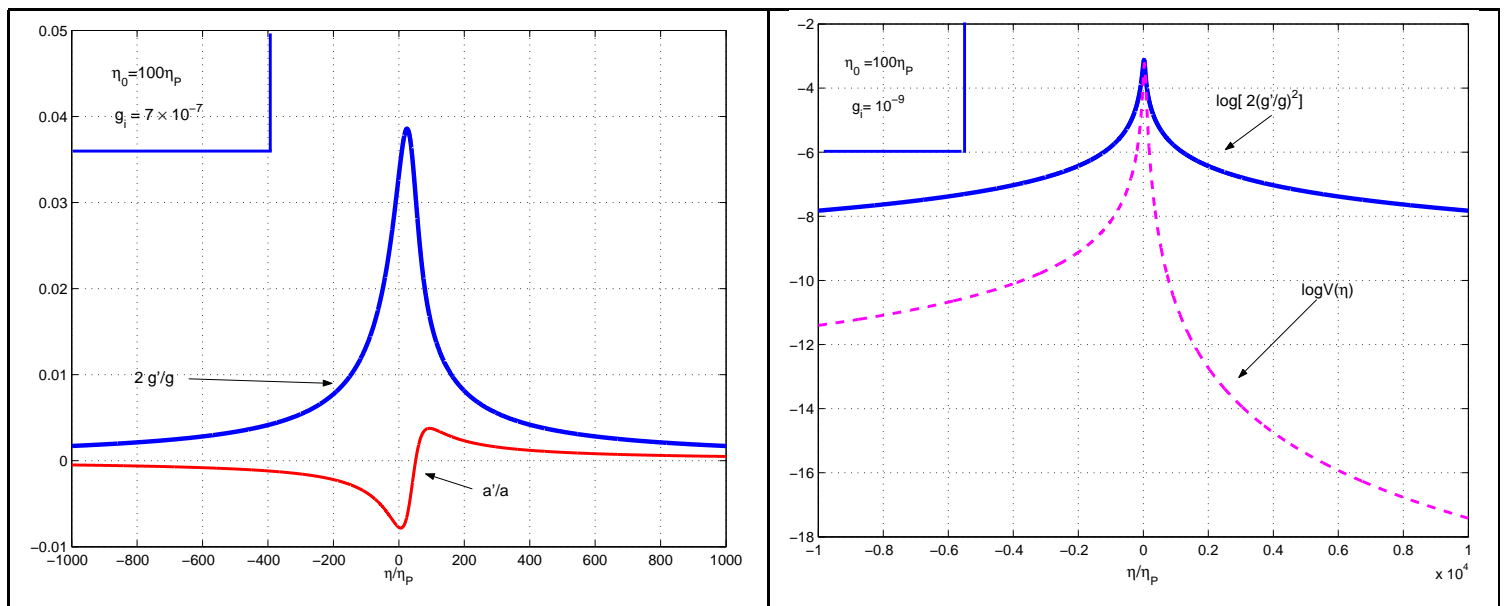

Figure 2.2: In the left plot cold-bounce solutions are illustrated in terms of $a^{\prime} / a$ and in terms of $g^{\prime} / g$. In the right plot the dilaton potential is compared with the dilaton kinetic term. The various quantities are indicated above or below each curve. As in Fig. 2.1, $\eta_{i}=-10^{-7} \eta_{\mathrm{P}}$ 
of 0.1 or 0.01 . In the present case, on the contrary, the dilaton coupling is always growing as a power of the conformal time after the bounce.

\section{Amplification of Abelian gauge bosons}

In the low-energy string effective action, massless Abelian gauge bosons are expected [20] as a result of the compactification procedure. The modes of the Abelian gauge bosons are super-adibatically amplified [8] and they re-enter, at different conformal times, after the bounce. The modes of comoving frequencies comparable with the typical curvature at the bounce will be the first ones to re-enter. Furthermore the high-frequency modes are also the most energetic and effectively behave like a fluid of high-frequency primordial photons.

Right at the bounce the energy density of the primordial photons is still small. However, as the geometry evolves, the energy density of the radiation fluid decreases slower than the energy density of the dilatonic sources and, therefore, after a transient time, a radiationdominated phase sets in. The typical transient time depends upon the maximal-curvature scale at the bounce and upon the number of massless species, which are parametrically amplified. Since in the present case only one Abelian gauge boson is present, the obtained estimates represent the minimal achievable efficiency of the process.

More specifically, consider the case where the coupling of the four-dimensional dilaton field to the gauge kinetic term is parametrized as

$$
S_{\mathrm{gb}}=-\frac{1}{4} \int d^{4} x \sqrt{-G} e^{-\varphi} F_{\alpha \beta} F^{\alpha \beta} .
$$

Defining the canonically normalized vector potential $A_{i}=g(\eta) \mathcal{A}_{i}$, in the radiation gauge

$$
\mathcal{A}_{0}=0, \quad \vec{\nabla} \cdot \overrightarrow{\mathcal{A}}=0,
$$

the canonical Lagrangian density can be obtained from Eq. ( 3.1) by dropping total derivatives:

$$
\mathcal{L}(\vec{x}, \eta)=\frac{1}{2}\left[\left(\partial_{\eta} \mathcal{A}_{i}\right)^{2}+\left(g^{-1}\right)^{\prime \prime} g \mathcal{A}_{i}^{2}-\left(\partial_{i} \mathcal{A}_{j}\right)^{2}\right],
$$

The two physical polarizations of the photon can be quantized according to the standard rules of quantization in the radiation gauge in curved space-times [17]:

$$
\hat{\mathcal{A}}_{i}(\vec{x}, \eta)=\sum_{\alpha} \int \frac{d^{3} k}{(2 \pi)^{3 / 2}}\left[\hat{a}_{k, \alpha} e_{i}^{\alpha} \mathcal{A}_{k}(\eta) e^{-i \vec{k} \cdot \vec{x}}+\hat{a}_{k, \alpha}^{\dagger} e_{i}^{\alpha} \mathcal{A}_{k}(\eta)^{\star} e^{i \vec{k} \cdot \vec{x}}\right],
$$

and

$$
\hat{\pi}_{i}(\vec{x}, \eta)=\sum_{\alpha} \int \frac{d^{3} k}{(2 \pi)^{3 / 2}}\left[\hat{a}_{k, \alpha} e_{i}^{\alpha} \Pi_{k}(\eta) e^{-i \vec{k} \cdot \vec{x}}+\hat{a}_{k, \alpha}^{\dagger} e_{i}^{\alpha} \Pi_{k}(\eta)^{\star} e^{i \vec{k} \cdot \vec{x}}\right]
$$


where $e_{i}^{\alpha}(k)$ describe the polarizations of the photon and

$$
\Pi_{k}(\eta)=\mathcal{A}_{k}^{\prime}(\eta), \quad\left[\hat{a}_{k, \alpha}, \hat{a}_{p, \beta}^{\dagger}\right]=\delta_{\alpha \beta} \delta^{(3)}(\vec{k}-\vec{p})
$$

The evolution equation for the mode functions will then be, in Fourier space,

$$
\mathcal{A}_{i}^{\prime \prime}+\left[k^{2}-g\left(g^{-1}\right)^{\prime \prime}\right] \mathcal{A}_{i}=0
$$

where, following the terminology of $[18,19]$ the "pump field" 3 can also be expressed as:

$$
g\left(g^{-1}\right)^{\prime \prime}=\left(\frac{\varphi^{\prime 2}}{4}-\frac{\varphi^{\prime \prime}}{2}\right)
$$

Equation ( 3.7) tells us that all the modes $k^{2}<\left|g\left(g^{-1}\right)^{\prime \prime}\right|$ are super-adiabatically amplified. Naively, the maximal amplified frequency will then be $k_{\max }^{2}=\left|g\left(g^{-1}\right)^{\prime \prime}\right|$. Since, for $\eta \rightarrow \pm \infty$, $g\left(g^{-1}\right)^{\prime \prime} \simeq \eta^{-2}$, modes with $k \eta>1$ are said to be inside the horizon, while modes $k \eta<1$ are outside the horizon. This not fully accurate terminology will also be employed in the following only for sake of simplicity. The maximally amplified modes are then the ones for which

$$
k_{\max }^{2} \simeq\left|g\left(g^{-1}\right)^{\prime \prime}\right|,
$$

as is illustrated directly in Fig. 3.3 for a particular choice of the parameters.

The Fourier modes appearing in Eq. ( 3.7) have to be normalized while they are inside the horizon for large and negative $\eta$. In this limit the initial conditions provided by quantum mechanics are

$$
\begin{aligned}
& \mathcal{A}_{k}(\eta)=\frac{1}{\sqrt{2 k}} e^{-i k \eta} \\
& \Pi_{k}(\eta)=-i \sqrt{\frac{k}{2}} e^{-i k \eta}
\end{aligned}
$$

In the limit $\eta \rightarrow+\infty$ the positive and negative frequency modes will be mixed, so that the solution will be represented in the plane wave orthonormal basis as

$$
\begin{aligned}
\mathcal{A}_{k} & =\frac{1}{\sqrt{2 k}}\left[c_{+}(k) e^{-i k \eta}+c_{-}(k) e^{i k \eta}\right], \\
\mathcal{A}_{k}^{\prime} & =-i \sqrt{\frac{k}{2}}\left[c_{+}(k) e^{-i k \eta}-c_{-}(k) e^{i k \eta}\right] .
\end{aligned}
$$

\footnotetext{
${ }^{3}$ Notice that the "pump field", i.e. the field transferring energy from the background to the fluctuations, is determined by the first and second derivatives of the gauge coupling.
} 


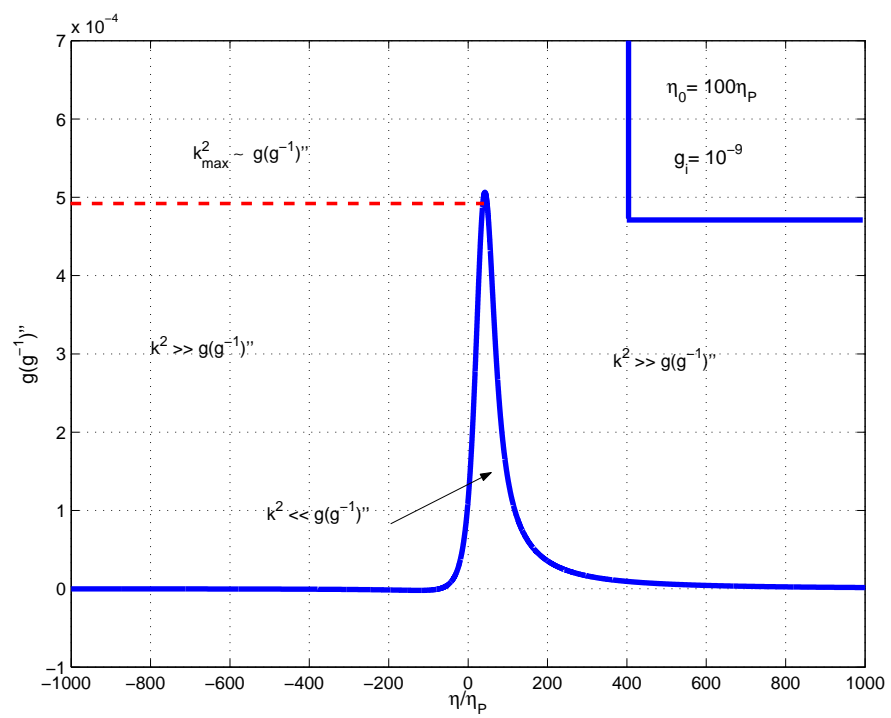

Figure 3.3: The pump field for the primordial photons (vertical axis) is plotted. For different values of $\eta_{0}$ the overall dimensions of the plot are rescaled (since $g\left(g^{-1}\right)^{\prime \prime} \sim \eta_{0}^{-2}$ ), but the relation determining $k_{\max } \eta_{0}$ always stays the same. In all the plots of the present section $\eta_{\mathrm{i}}=-10^{7} \eta_{\mathrm{P}}$

where $c_{ \pm}(k)$ are the (constant) mixing coefficients. The following two relations fully determine the square modulus of each of the two mixing coefficients in terms of the complex wave-functions obeying Eq. ( 3.7$)$ :

$$
\begin{aligned}
& \left|c_{+}(k)\right|^{2}-\left|c_{-}(k)\right|^{2}=i\left(\mathcal{A}_{k}^{\star} \Pi_{k}-\mathcal{A}_{k} \Pi_{k}^{\star}\right), \\
& \left|c_{+}(k)\right|^{2}+\left|c_{-}(k)\right|^{2}=\frac{1}{k^{2}}\left(\left|\Pi_{k}\right|^{2}+k^{2}\left|\mathcal{A}_{k}\right|^{2}\right) .
\end{aligned}
$$

After having numerically computed the time evolution of the properly normalized mode functions, Eqs. ( 3.12) and (3.13) can be used to infer the value of the relevant mixing coefficient (i.e. $c_{-}(k)$ ). Equation ( 3.12$)$ is, in fact, the Wronskian of the solutions. If the second-order differential equation is written in the form (3.7), the Wronskian is always conserved throughout the time evolution of the system. Since, from Eq. ( 3.10), the Wronskian is equal to 1 initially, it will be equal to 1 all along the time evolution. Thus, from Eq. ( 3.12) $\left|c_{+}(k)\right|^{2}=\left|c_{-}(k)\right|^{2}+1$. The fact that the Wronskian must always be equal to 1 is the measure of the precision of the algorithm.

In Fig. 3.4 the numerical calculation of the spectrum is illustrated for different values of $k \eta_{0}$. In the left plot the mixing coefficients are reported for modes $k \ll k_{\max }$. In the right plot the mixing coefficients are reported for modes around $k_{\max }$. Clearly, from the left plot a smaller $k$ leads to a larger mixing coefficient which means that the spectrum is 
rather blue. Furthermore by comparing the amplification of different modes it is easy to infer that the scaling law is $\left|c_{+}(k)\right|^{2}+\left|c_{-}(k)\right|^{2} \propto\left(k / k_{\max }\right)^{-n_{g}}$, with $n_{g} \sim 3.46$, which is in excellent agreement with the analytical determination of the mixing coefficients leading to $n_{g}=2 \sqrt{3} \sim 3.46$ [see below, Eq. (3.23)].

The second piece information that can be drawn from Fig. 3.4 concerns $k_{\max }$, whose specific value

$$
k_{\max } \simeq \frac{\sqrt{5}-0.5}{\eta_{0}}
$$

can be determined numerically for different values of $\eta_{0}$ and will be important for an accurate estimate of the back-reaction effects.

A reasonably accurate estimate of the maximal amplified frequency is necessary in order to match the analytical estimates with the numerical calculations of the back-reaction effects. For the value of $k_{\max }$ reported in Eq. (3.14), the obtained mixing coefficient is 1 , i.e. $\left|c_{-}\left(k_{\max }\right)\right| \simeq 1$. According to Fig. 3.4 (right plot) as we move from $k_{\max }$ to larger $k$, $\left(\left|c_{+}(k)\right|^{2}+\left|c_{-}(k)\right|^{2}\right) \simeq\left(\left|c_{+}(k)\right|^{2}-\left|c_{-}(k)\right|^{2}\right)$ implying that $\left|c_{-}(k)\right| \sim 0$. Moreover, from the left plot of Fig. 3.4 it can be appreciated that

$$
\left|c_{-}\left(k_{\max }\right)\right|^{2}=1, \quad \log \left(\left|c_{+}\left(k_{\max }\right)\right|^{2}+\left|c_{-}\left(k_{\max }\right)\right|^{2}\right)=\log 3 \simeq 0.477 .
$$

Thus the absolute normalization and slope of the relevant mixing coefficient can be numerically determined to be

$$
\left|c_{-}(k)\right|^{2}=\left(\frac{k}{k_{\max }}\right)^{-2 \sqrt{3}} .
$$

In Fig. 3.5 (left plot), the predictions of Eq. ( 3.16) at different $k$ modes are compared with the numerical calculation. It can be concluded that Eq. ( 3.16) is rather accurate as far as both the slope and the absolute normalization are concerned.

The numerical estimates presented so far can be also corroborated by the usual analytical treatment based on the matching of the solutions for the mode functions before and after the bounce. The evolution of the modes described by Eq. ( 3.7) can be approximately determined from the exact asymptotic solutions given in Eqs. (2.32) and (2.33), and implying that $\varphi_{ \pm}^{\prime} \simeq \pm \sqrt{3} / \eta$. Thus the solutions of Eq. ( 3.7$)$ can be obtained in the two asymptotic regimes

$$
\begin{aligned}
& \mathcal{A}_{k,-}(\eta)=\frac{\sqrt{-\pi \eta}}{2} e^{i \frac{\pi}{2}(\nu+1 / 2)} H_{\nu}^{(1)}(-k \eta), \quad \eta \leq-\eta_{1} \\
& \mathcal{A}_{k,+}(\eta)=\frac{\sqrt{\pi \eta}}{2} e^{i \frac{\pi}{2}(\mu+1 / 2)}\left[c_{-} H_{\mu}^{(1)}(k \eta)+c_{+} e^{-i \pi(\mu+1 / 2)} H_{\mu}^{(2)}(k \eta)\right], \eta \geq-\eta_{1}
\end{aligned}
$$

where $H_{\alpha}^{(1,2)}$ are Hankel functions of first and second kind [21] whose related indices are

$$
\nu=\frac{\sqrt{3}-1}{2}, \quad \mu=\frac{\sqrt{3}+1}{2} \text {. }
$$




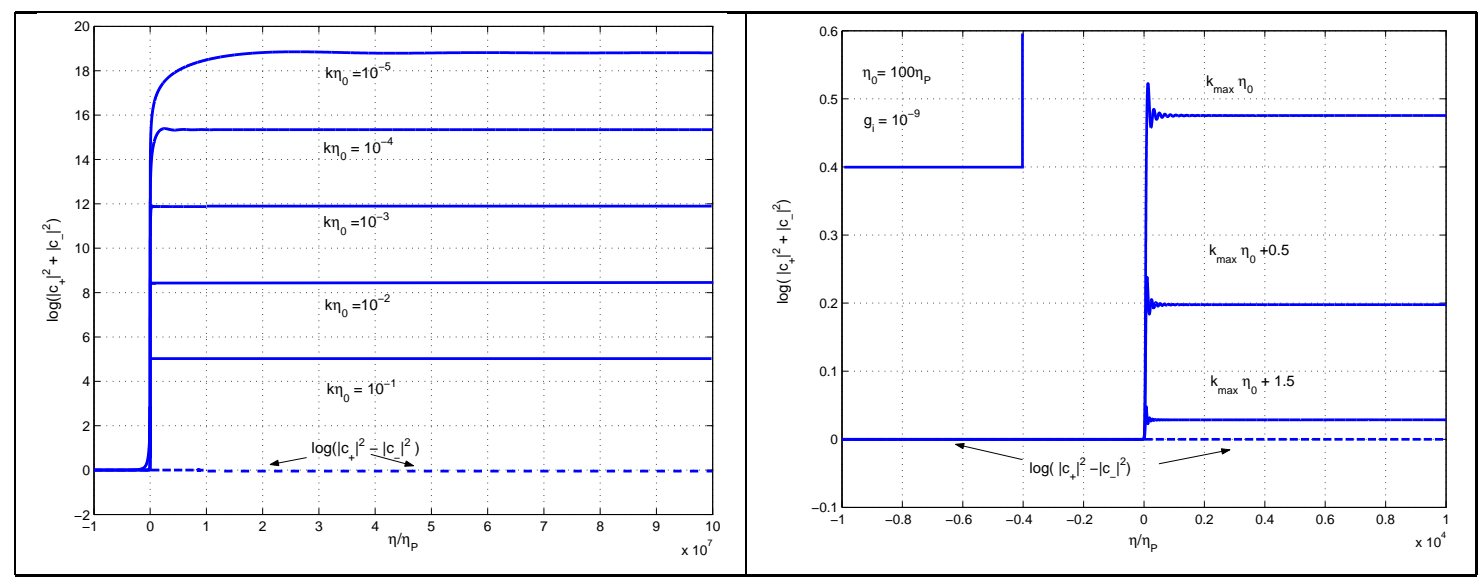

Figure 3.4: In the left plot the amplification of modes $k^{2} \ll\left|g\left(g^{-1}\right)^{\prime \prime}\right|$ is illustrated. The time evolution of the pump field $g\left(g^{-1}\right)^{\prime \prime}$ was described in Fig. 3.3. In the right plot the largest amplified $k$, i.e. $k \simeq k_{\max }$, are discussed. The specific value of $k_{\max }$ discussed in Eq. (3.14) leads to $\mid c_{-}\left(k_{\max }\right) \simeq 1$.

The time scale $\eta_{1}$ defines the width of the bounce and, typically, $\eta_{1} \sim \eta_{0}$.

Phases appearing in Eq. ( 3.17$)$ are carefully chosen so that

$$
\lim _{\eta \rightarrow-\infty} \mathcal{A}_{k}=\frac{1}{\sqrt{2 k}} e^{-i k \eta}
$$

Using then the appropriate matching conditions

$$
\begin{aligned}
& \mathcal{A}_{k,-}\left(-\eta_{1}\right)=\mathcal{A}_{k,+}\left(\eta_{1}\right), \\
& \mathcal{A}_{k,-}^{\prime}\left(-\eta_{1}\right)=\mathcal{A}_{k,+}^{\prime}\left(\eta_{1}\right),
\end{aligned}
$$

and defining $x_{1}=k \eta_{1}$, the obtained mixing coefficients are

$$
\begin{aligned}
& c_{+}(k)=i \frac{\pi}{4} x_{1} e^{i \pi(\nu+\mu+1) / 2}\left[-\frac{\nu+\mu+1}{x_{1}} H_{\mu}^{(1)}\left(x_{1}\right) H_{\nu}^{(1)}\left(x_{1}\right)\right. \\
& \left.+H_{\mu}^{(1)}\left(x_{1}\right) H_{\nu+1}^{(1)}\left(x_{1}\right)+H_{\mu+1}^{(1)}\left(x_{1}\right) H_{\nu}^{(1)}\left(x_{1}\right)\right], \\
& c_{-}=i \frac{\pi}{4} x_{1} e^{i \pi(\nu-\mu) / 2}\left[-\frac{\nu+\mu+1}{x_{1}} H_{\mu}^{(2)}\left(x_{1}\right) H_{\nu}^{(1)}\left(x_{1}\right)\right. \\
& \left.+H_{\mu}^{(2)}\left(x_{1}\right) H_{\nu+1}^{(1)}\left(x_{1}\right)+H_{\mu+1}^{(2)}\left(x_{1}\right) H_{\nu}^{(1)}\left(x_{1}\right)\right],
\end{aligned}
$$

satisfying the exact Wronskian normalization condition $\left|c_{+}(k)\right|^{2}-\left|c_{-}(k)\right|^{2}=1$. In the small argument limit, i.e. $k \eta_{1} \sim k \eta_{0} \ll 1$ the leading term in Eq. ( 3.22$)$ leads to

$$
c_{-}(k) \simeq \frac{i 2^{\mu+\nu}}{4 \pi} e^{i \pi(\nu-\mu) / 2} x_{1}^{-\mu-\nu}(\nu+\mu-1) \Gamma(\mu) \Gamma(\nu)
$$




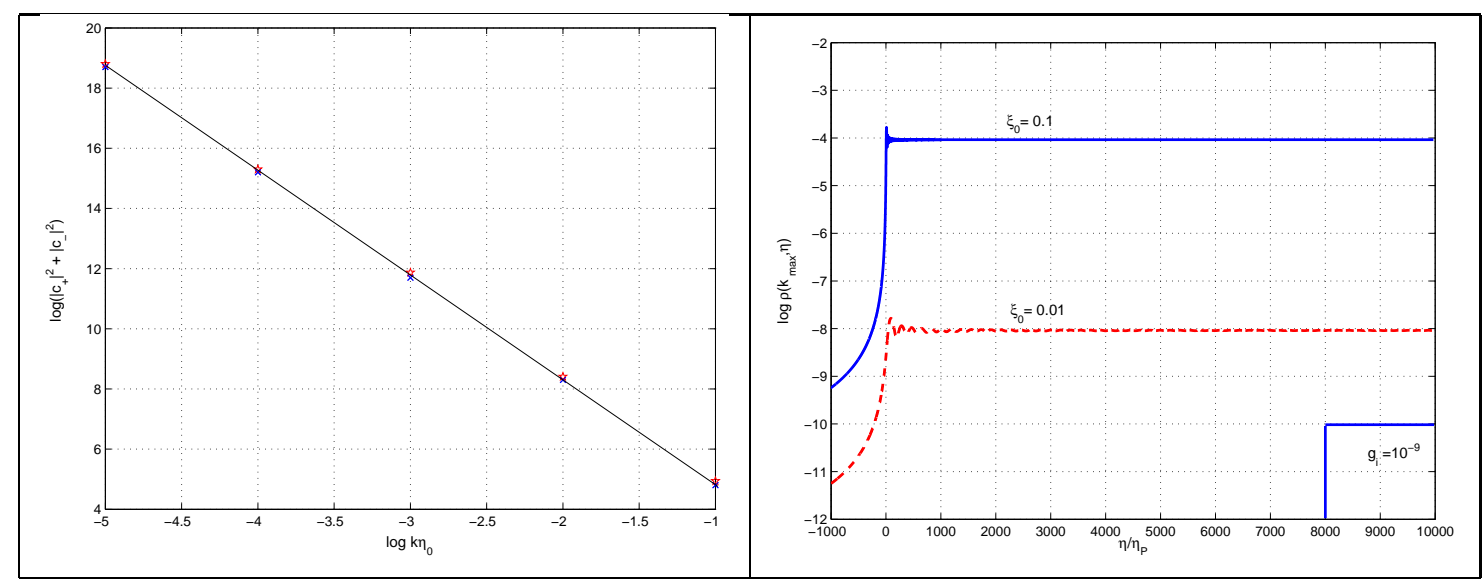

Figure 3.5: In the left plot the mixing coefficient computed from Eq. ( 3.16) (crosses) are compared with the numerical results (stars). The difference is barely visible. In the right plot the logarithmic energy spectrum is computed for $k=k_{\max }$ and for different sets of parameters. The quantity $\xi_{0}=\mathcal{H}_{0} / M_{\mathrm{P}}$ has been introduced.

If we now insert the values given in Eq. (3.18) it turns out that $c_{-}(k) \simeq 0.41\left|k \eta_{1}\right|^{-\sqrt{3}}$. The spectral slope agrees with the numerical estimate, as already stressed. The absolute normalization cannot be determined from Eq. ( 3.23), where the small argument limit has already been taken. In order to determine the absolute normalization the specific value of $k_{\max } \eta_{1}$ has to be inserted in Eq. ( 3.22$)$. The result of this procedure, taking $\eta_{1} \sim \eta_{0}$ is $\left|c_{-}\left(k_{\max }\right)\right|^{2}=0.14$, which is roughly a factor of 10 smaller than the interpolating formula given in Eq. ( 3.16$)$.

\section{Gravitational reheating of a cold bounce}

A reasonably accurate determination of the energy density of the modes re-entering the horizon after the bounce and modifying the dynamics of the background can now be presented. The expression of the canonical energy-momentum tensor derived from the action ( 3.1$)$ is

$$
T_{\mu}^{\nu}=e^{-\varphi}\left[-F_{\mu \alpha} F^{\nu \alpha}+\frac{\delta_{\mu}^{\nu}}{4} F_{\alpha \beta} F^{\alpha \beta}\right] .
$$

From the explicit expression of Eq. (4.1) in terms of the gauge potentials $\mathcal{A}_{i}$, it is possible to derive the expectation values of the various components of the canonical energy-momentum tensor. While the derivation of these results is included in the Appendix, the relevant results, 
for the present calculation, are

$$
\begin{aligned}
& \rho_{\mathrm{r}}=\left\langle T_{0}^{0}(\eta)\right\rangle=\frac{1}{a^{4}} \int d \ln k \rho(k, \eta), \\
& \rho(k, \eta)=\frac{k^{3}}{2 \pi^{2}}\left\{\left|\Pi_{k}\right|^{2}+\left[\left(\frac{g^{\prime}}{g}\right)^{2}+k^{2}\right]\left|\mathcal{A}_{k}\right|^{2}+\frac{g^{\prime}}{g}\left[\Pi_{k}^{*} \mathcal{A}_{k}+\Pi_{k} \mathcal{A}_{k}^{*}\right]\right\}
\end{aligned}
$$

and

$$
\left\langle T_{i}^{j}(\eta)\right\rangle=-\frac{\rho_{\mathrm{r}}}{3} \delta_{i}^{j}, \quad\left\langle T_{i}^{0}(\eta)\right\rangle=0
$$

Sometimes $\rho(k, \eta)$ is called the logarithmic energy spectrum, since it gives the energy density of the field per logarithmic interval of frequency.

For $\eta \rightarrow-\infty$ and $|k \eta|>1$ (i.e. when the modes are inside the horizon at the onset of the dynamical evolution) the exact mode functions given in Eq. ( 3.17) imply that the logarithmic energy spectrum is

$$
\rho(k, \eta)=\frac{k^{4}}{4 \pi^{2}}\left[2+\frac{3}{k^{2} \eta^{2}}+O\left(\frac{1}{|k \eta|^{4}}\right)\right] .
$$

The first term is nothing but the energy density of the initial vacuum state that can be subtracted. The remaining terms are suppressed by powers of $|k \eta|^{-1}$ and are negligible in this regime.

For $|k \eta|<1$ the modes are super-adiabatically amplified. In this case the logarithmic energy density becomes, from Eqs. ( 3.17 ) and (4.3)

$$
\rho(k, \eta)=\frac{k^{4}}{8 \pi}(-k \eta)\left[\left|H_{\nu+1}^{(1)}(-k \eta)\right|^{2}+\left|H_{\nu}^{(1)}(-k \eta)\right|^{2}\right] .
$$

Expanding for $|k \eta|<1$, and recalling that Eq. (4.6) is valid for negative $\eta$, the leading term is

$$
\rho(k, \eta)=\frac{k^{4}}{8 \pi^{3}} \Gamma^{2}(\nu+1) 2^{2 \nu+2}(-k \eta)^{-2 \nu-1},
$$

where $2 \nu+1=\sqrt{3}$.

Finally, after the bounce and when the modes have re-entered the horizon, the logarithmic energy density becomes

$$
\begin{aligned}
\rho(k, \eta)= & \frac{k^{4}}{4 \pi^{2}}\left[2\left|c_{+}(k)\right|^{2}+2\left|c_{-}(k)\right|^{2}\right. \\
& +\frac{3}{k^{2} \eta^{2}}\left(\left|c_{+}(k)\right|^{2}+\left|c_{-}(k)\right|^{2}+c_{+}^{*}(k) c_{-}(k) e^{2 i k \eta}+c_{-}^{*} c_{+} e^{-2 i k \eta}\right) \\
& \left.+i \frac{\sqrt{3}}{k \eta}\left(c_{+}^{*}(k) c_{-}(k) e^{2 i k \eta}-c_{-}^{*}(k) c_{+}(k) e^{-2 i k \eta}\right)\right] .
\end{aligned}
$$

This expression also contains subleading terms going as higher powers of $|k \eta|^{-1}$. However, these terms are negligible when the modes have already re-entered. Hence, using the relation 
between the mixing coefficients, the leading term appearing in Eq. (4.8) can be rewritten as

$$
\rho(k, \eta) \simeq \frac{k^{4}}{\pi^{2}}\left|c_{-}(k)\right|^{2},
$$

where the initial zero-point energy has already been subtracted. Notice that this final estimate is consistent with the interpretation of $\left|c_{-}(k)\right|^{2}$ as the mean number of produced particles per phase-space interval.

With this analytical understanding, we are then ready to discuss in detail the effect of the produced small-scale fluctuations on the dynamics of the background geometry. As expected from the vanishing trace of Eq. (4.1), the effective relation between the components of the averaged canonical energy-momentum tensor leads to $3 p_{\mathrm{r}}=\rho_{\mathrm{r}}$ (see also Appendix B). Thus the effect of $\rho_{\mathrm{r}}$ as given in Eq. (4.2) should be inserted back into Eqs. (2.26) and (2.27) with the result that, for instance, the following equation can be obtained:

$$
\mathcal{H}^{\prime}=-\frac{2}{3}\left(\frac{g^{\prime}}{g}\right)^{2}+\frac{5}{6} \frac{V_{0}}{g^{2} a^{10}}-\frac{a^{2}}{6} \rho_{\mathrm{r}}
$$

In the numerical code, Eq. (4.10) is consistently solved together with the other equations for the dilaton and for the fluctuations of the gauge fields. The problem is simplified by the following observation. The integration over $k$ appearing in Eq. (4.2) runs up to $k_{\max }$. Since the logarithmic energy spectrum decreases at large-distance scales, the integral

$$
\rho_{\mathrm{r}}(\eta)=\frac{1}{a^{4}(\eta)} \int^{k_{\max }} \rho(k, \eta) \frac{d k}{k}
$$

is well estimated by the value of the logarithmic energy spectrum at $k_{\max }$ :

$$
\rho_{\mathrm{r}}(\eta)=\frac{k_{\max }^{4}}{a^{4}(\eta)} \epsilon_{0}
$$

where $\epsilon_{0}=0.19$.

The result of eq. (4.12) reproduces extremely well the numerical results reported, for instance, in Fig. 3.5 (right plot) if we recall the explicit form of $k_{\max }$ given in the previous section.

The numerical integration may still be rather stiff because of the different scales present in the model (in particular the width of the bounce may be much smaller, for instance, than the typical time at which radiation becomes dominant). For this purpose the Rosenbrok method (particularly suitable for stiff problems [22]) has been used after the bounce.

Before an illustration of the numerical results, the main semi-analytical expectations will be discussed. The typical time scale defining the dominance of radiation can be predicted by estimating, numerically, the time of re-entry of $k_{\max }$ and by comparing the relative balance 
between the energy density of the background and the energy density of the fluctuations. The time of re-entry of $k_{\max }$ is

$$
\eta_{\max } \sim \frac{1}{k_{\max }} \sim \frac{1}{\epsilon_{1}} \eta_{0}
$$

where $\epsilon_{1} \sim 1.73$.

From this expression, assuming that after the bounce the background is still dominated by the kinetic energy of the dilaton, the time $\eta_{*}$ can be estimated to be

$$
\frac{\eta_{*}}{\eta_{\mathrm{P}}} \simeq \frac{\epsilon_{2}}{\epsilon_{0}}\left(\frac{H_{0}}{M_{\mathrm{P}}}\right)^{-3}
$$

where $\epsilon_{2} \sim 12.9$. For instance, for $H_{0} / M_{\mathrm{P}} \sim 10^{-2}$, and given the indetermination on the numerical parameters, $\eta_{*} \simeq 6 \times 10^{7} \eta_{\mathrm{P}}$.

After $\eta_{*}$ the evolution is expected to be dominated by radiation, which means that the scale factor should go linearly with the conformal time. During radiation the potential term becomes even more negligible than in the case of a cold-bounce solution, where it was already negligible (recall Fig. 2.2, right plot). Hence, the evolution of the dilaton will be, from the background equation (2.31),

$$
\left(a^{2} \varphi^{\prime}\right)^{\prime}=0
$$

which also implies that

$$
\varphi \simeq \varphi_{1}-\varphi_{2}\left(\frac{\eta_{\mathrm{P}}}{\eta}\right)
$$

where $\varphi_{1}$ and $\varphi_{2}$ are integration constants.

A useful function, which can be used as a diagnostic of a fully achieved transition to radiation, is

$$
z(\eta)=\frac{a \varphi^{\prime}}{\mathcal{H}} .
$$

It should be noticed that $z(\eta) \sim a(\eta)$, if evaluated on the cold-bounce solution, while $z(\eta)$ is strictly constant, in the case of a radiation-dominated background, by virtue of Eqs. (4.15) and (4.16).

The analytical expectations suggested by Eqs. (4.14)-(4.17) are confirmed by the numerical integration of the full system, which is illustrated in Figs. 4.6 and 4.7. The constancy of $z(\eta)$ is exactly what is numerically observed. In Fig. 4.6 (left plot), after a typical timescale $\eta_{*} \sim 10^{6} \eta_{\mathrm{P}}, z(\eta)$ becomes constant while it should still increase as $z(\eta) \sim a(\eta) \simeq \sqrt{\eta}$ if the Universe would not be dominated by radiation. At the same time (Fig. 4.6, right plot) the scale factor grows linearly and (Fig. 4.7, left plot) the dilaton goes to constant, as implied by Eq. (4.16). 

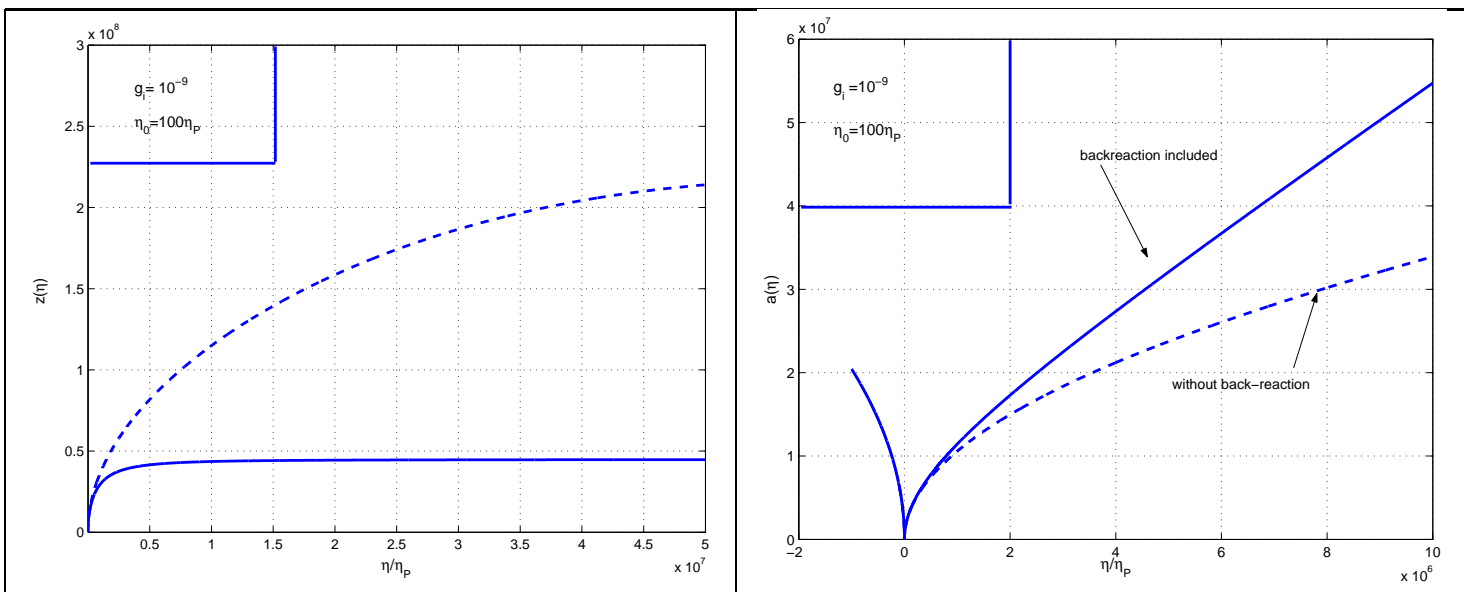

Figure 4.6: In the left plot the evolution of $z(\eta)$, defined in Eq. (4.17), is illustrated. This simple function is a diagnostic of a fully achieved transition to radiation where it is constant, while it should grow as $\sqrt{\eta}$ (dotted line) in the case when the back-reaction of photons is not included. In the right plot the evolution of the scale factor is illustrated with (solid line) and without (dashed line) back-reaction effects.
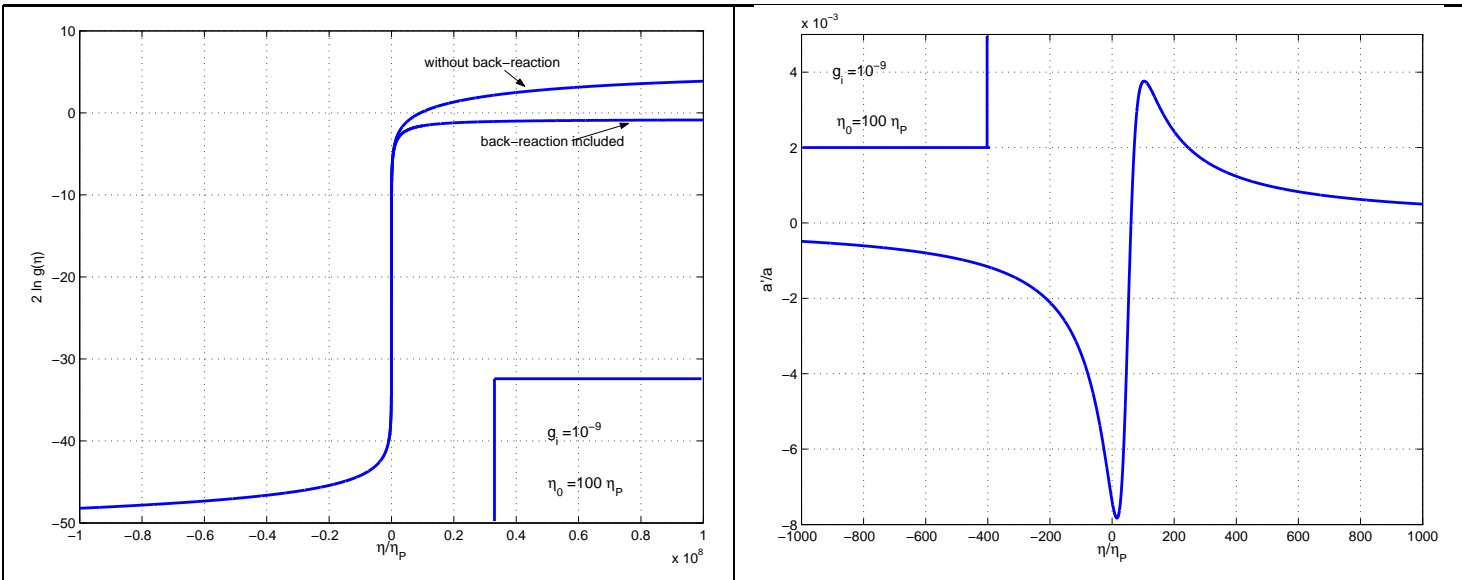

Figure 4.7: In the left plot the evolution of the logarithm of the gauge coupling is illustrated. In the right plot the evolution of $a^{\prime} / a$ is presented. 


\section{Concluding discussions}

Cold-bounce solutions have virtues and problems. Their main virtues are that they lead to computable models of the bouncing dynamics at low curvatures. The main problems are related to the fact that the Universe emerging after the bounce is rather cold and dominated by the dilaton. This occurrence also implies that the dilaton will always be growing, before and after the bounce.

In the present paper a model of gravitational heating of the cold bounce has been proposed. The model considers only the effect of one species of Abelian gauge bosons, which are super-adiabatically amplified. The frequencies that are maximally amplified are comparable with the typical curvature of the Universe at the bounce and effectively behave like a fluid of primordial photons. The following results have been illustrated:

- an accurate numerical method for the calculation of the amplification of the primordial photons has been developed;

- taking into account the back-reaction of the primordial photons, the cold-bounce solution can be consistently heated;

- the transition to radiation can occurr for sub-Planckian curvature scales;

- solutions have been presented where the dilaton goes to a constant value and the asymptotic value of the gauge coupling is always smaller than 1.

\section{Acknowledgements}

The author wishes to thank G. Veneziano for useful discussions. 


\section{A Solutions with and without fluid sources}

Equations (2.10)-(2.13) can be written in generic $d$ spatial dimensions as:

$$
\begin{aligned}
& \dot{\bar{\varphi}}^{2}-d H^{2}-V=e^{\bar{\varphi}} \bar{\rho}, \\
& \dot{H}=\dot{\varphi} H+\frac{1}{2} e^{\bar{\varphi}} \bar{p}, \\
& 2 \ddot{\bar{\varphi}}-\dot{\bar{\varphi}}^{2}-d H^{2}+V-\frac{\partial V}{\partial \bar{\varphi}}=0, \\
& \dot{\bar{\rho}}+d H \bar{p}=0 .
\end{aligned}
$$

In this Appendix, cold-bounce solutions with and without fluid sources will be presented. It will be shown that the naive addition of fluid sources does not stabilize the evolution of the dilaton field after the bounce. Since throughout this Appendix we will always consider solutions in the string frame, the subscript has been omitted in the quantities appearing in Eqs. (A.1)-(A.4).

\section{A.1 Solutions without fluid sources}

When fluid sources are absent there is a class of regular solutions in the presence of exponential potential of the form $V(\bar{\varphi})=-V_{0} e^{\alpha \bar{\varphi}}$. Defining the appropriate variables of the phase space

$$
u=\frac{\dot{\varphi}}{\sqrt{d} H}, \quad v=\frac{\sqrt{-V}}{\sqrt{d} H},
$$

the constraint (A.1) becomes $u^{2}+v^{2}=1$ and the equations for $u$ and $v$ are

$$
\begin{aligned}
& \frac{d u}{d \tau}=\sqrt{d}\left(1-u^{2}\right)-\frac{\alpha}{2} \sqrt{d} v^{2} \equiv-\frac{\sqrt{d}}{2}(\alpha-2)\left(1-u^{2}\right), \\
& \frac{d v}{d \tau}=\sqrt{d}\left(\frac{\alpha}{2}-1\right) u v
\end{aligned}
$$

where, in the first equation, the equality follows from $u^{2}+v^{2}=1$ and where $\tau=\log a$. Eqs. (A.6) and (A.7) then are in the form of an autonomous system in the plane $(u, v)$. From Eq. (A.5) it is clear that

$$
t \rightarrow-t, \quad u \rightarrow u, \quad v \rightarrow-v .
$$

Hence, the system (A.6)-(A.7) is symmetric for $u \rightarrow u$ and $v \rightarrow-v$ when $\tau \rightarrow-\tau$. Thanks to the above symmetry the phase space of the autonomous system (A.6)-(A.7) is fully characterized by half of the unit disk, for instance, the one for $v \geq 0$.

The critical points of the system are given by

$$
u_{c}= \pm 1, \quad v_{c}=0 .
$$


The plane autonomous system can then be linearized around the critical points given in Eq. (A.9), namely

$$
\begin{aligned}
& u=u_{c}+\epsilon_{u}, \quad v=v_{c}+\epsilon_{v}, \\
& \frac{d \epsilon_{u}}{d \tau}=2 \mu u_{c} \epsilon_{u}, \\
& \frac{d \epsilon_{v}}{d \tau}=\mu u_{c} \epsilon_{v}
\end{aligned}
$$

where $2 \mu=\sqrt{d}(\alpha-2)$. If $u_{c}=1$ the critical point is an unstable node since the two eigenvalues have the same (positive) sign. If $u_{c}=-1$ the critical point is a stable node since the two eigenvalues have the same sign and are both negative. The unstable node $(1,0)$ corresponds to the accelerated branch of the solution where the dilaton coupling increases and the curvature scale also increases. The stable node $(-1,0)$ corresponds to the dual (decelerated) solution with decreasing dilaton and decreasing curvature.

In this parametrization the evolution equations can be easily integrated. From Eq. (A.6), integrating once and inverting the obtained expression:

$$
u(\tau)=\frac{1+\mathcal{C} e^{2 \mu \tau}}{1-\mathcal{C} e^{2 \mu \tau}}
$$

where the integration constant $\mathcal{C}$ has to be selected such that the appropriate boundary conditions are satisfied. The constant $\mathcal{C}$ can be chosen in such a way that $u(\tau)$ is everywhere regular in its domain of definition and, in particular, for $\tau \rightarrow 0$. With this in mind, $\mathcal{C}=-1$ in order to prevent a possible singularity in $\tau=0$. From Eqs. (A.7) and (A.13), the full result is

$$
\begin{aligned}
& u(\tau)=-\tanh \mu \tau, \\
& v(\tau)=\frac{1}{\cosh \mu \tau},
\end{aligned}
$$

where the possible further integration constant is fixed by the constraint $u^{2}+v^{2}=1$.

The solution given by Eqs. (A.14) and (A.15) interpolates between the unstable node $(1,0)$ and the stable node $(-1,0)$, as is apparent from the asymptotic behaviour of $(u, v)$ for $\tau \rightarrow \pm \infty$. Recalling now the explicit definition of $u$ and $v$ in terms of the original background fields, i.e. Eqs. (A.6) and (A.7), and recalling the explicit parametrization of the exponential potential, the following chain of identities can be obtained:

$$
\begin{aligned}
& u(\tau)=\frac{\dot{\bar{\varphi}}}{\sqrt{d} H} \equiv \frac{1}{\sqrt{d}} \frac{d \bar{\varphi}}{d \tau}=-\tanh \mu \tau, \\
& v(\tau)=\frac{\sqrt{V_{0}} e^{\frac{\alpha}{2} \bar{\varphi}}}{\sqrt{d} H}=\frac{1}{\cosh \mu \tau} .
\end{aligned}
$$


Integrating once Eq. (A.16) and substituting in (A.17):

$$
\begin{aligned}
& H(\tau)=\frac{\sqrt{V_{0}}}{\sqrt{d}} \frac{e^{\frac{\alpha}{2} \bar{\varphi}}}{(\cosh \mu \tau)^{2 /(\alpha-2)}}, \\
& \bar{\varphi}(\tau)=\varphi_{0}-\frac{2}{\alpha-2} \ln \cosh (\mu \tau) .
\end{aligned}
$$

The parametrization in terms of $\tau=\ln a$ is then more useful than the one in terms of $t$. In fact, Eqs. (A.18) and (A.19) have simple analytical expressions in terms of $\tau$, but not necessarily in terms of $t$. Recalling that, by definition, $d \tau / d t=H(\tau)$, the explicit relation between $\tau$ and $t$ can be worked out by integrating once $H(\tau)$ from Eq. (A.18). For instance, in the case $\alpha=4$

$$
\begin{aligned}
& \bar{\varphi}=\varphi_{0}-\ln \cosh \sqrt{3} \tau, \\
& \frac{d \tau}{d t}=\frac{e^{2 \varphi_{0}} \sqrt{V_{0}}}{\sqrt{3} \cosh \sqrt{3} \tau},
\end{aligned}
$$

defining $t_{0}^{-1}=e^{2 \varphi_{0}} \sqrt{V_{0}}$,

$$
t / t_{0}=\sinh \sqrt{3} \tau
$$

i.e. $\tau=\ln a=(1 / \sqrt{3}) \ln \left[\left(t / t_{0}\right)^{2}+\sqrt{\left(t / t_{0}\right)^{2}+1}\right]$. This last solution is the one quoted in Eqs. (2.15)-(2.17). Note that if we do not want $H(\tau)$ blowing up for $|\tau| \rightarrow \infty$, we have

to demand $\alpha \geq 2$. In the case $\alpha=2, \mu=0$ and Eqs. (A.6)-(A.7) imply that $u$ and $v$ are constant.

\section{A.2 Solutions with fluid sources}

If fluid sources are included, various solutions are possible. Consider first the case $p=0$. In this case Eq. (2.11) can be immediately integrated and an exact regular solution of the remaining equations is

$$
V=-V_{0} e^{\bar{\varphi}}-V_{1} e^{4 \bar{\varphi}}
$$

with

$$
\begin{aligned}
& H=\frac{1}{\sqrt{d}} \frac{t_{0}}{\sqrt{t^{2}+t_{0}^{2}}}, \\
& \bar{\varphi}=\varphi_{0}-\frac{1}{2} \log \left[\frac{t^{2}}{t_{0}^{2}}+1\right], \\
& \rho=\bar{\rho}_{0} a^{-d},
\end{aligned}
$$

subject to the conditions:

$$
\begin{aligned}
& \bar{\rho}_{0}=V_{0}, \\
& V_{1} e^{2 \varphi_{0}}=\frac{1}{t_{0}^{2}} .
\end{aligned}
$$


Consider now the case where the barotropic index

$$
w=\frac{p}{\rho}=\frac{\bar{p}}{\bar{\rho}}
$$

is constant, and the dilaton potential is $V=-V_{0} e^{2 \bar{\varphi}}$. In this case, Eqs. (A.2) and (A.3) can be integrated :

$$
\begin{aligned}
& H=w \frac{e^{\bar{\varphi}}}{L}\left(x+x_{1}\right), \\
& \dot{\bar{\varphi}}=-\frac{e^{\frac{\bar{\varphi}}{L}}}{L}\left(x+x_{0}\right),
\end{aligned}
$$

where

$$
\frac{d x}{d t}=\frac{L}{2} \bar{\rho}
$$

and $x_{1}, x_{0}$ are integration constants ${ }^{4}$.

From Eq. (A.26) it can be checked that $\dot{\varphi}=\dot{\bar{\varphi}}+3 H$ is always positive only in the case $w=1 / 3$, corresponding to the case of a radiation fluid. In this case, an appropriate choice of the parameters $x_{1}$ and $x_{0}$ allows a simple analytical solution, which can be expressed directly in terms of $x$, i.e. the new time variable. If $w=1 / 3$, then Eq. (A.26) can be inserted back into Eq. (A.1), with the result

$$
e^{\bar{\varphi}}=\frac{3 \bar{\rho} L^{2}}{3\left(x+x_{0}\right)^{2}-\left(x+x_{1}\right)^{2}+3 V_{0} L^{2}} .
$$

Now we can choose constants to further simplify the general solution. Choose, in particular,

$$
x_{0}=L, \quad x_{1}=3 x_{0}, \quad V_{0} L^{2}=\frac{8}{3} L^{2} .
$$

Then, it can be checked that the explicit solution is, in this case

$$
\begin{aligned}
& a(x)=\sqrt{x^{2}+L^{2}} e^{3 \arctan (x / L)}, \\
& \frac{a^{\prime}}{a}=\frac{x+3 L}{x^{2}+L^{2}}, \\
& \bar{\varphi}^{\prime}=-\frac{3(x+L)}{x^{2}+L^{2}}, \\
& \frac{d x}{d t}=\frac{\rho_{0} L}{2} \frac{1}{a(x)} .
\end{aligned}
$$

The shifted dilaton is

$$
\bar{\varphi}=\varphi_{0}-3 \arctan (x / L)-\frac{3}{2} \ln \left(x^{2}+L^{2}\right) .
$$

\footnotetext{
${ }^{4}$ Since $\bar{\rho}$ has dimensions of an inverse length, $x$ and $t$ have the same dimensions.
} 
Looking at the constraint, we should also bear in mind that

$$
e^{\varphi_{0}}=\frac{3}{2} \bar{\rho}_{0} L^{2}
$$

There is a third class of possible solutions of the system of Eqs. (2.10)-(2.13). This class corresponds to the case when the barotropic index is a function of time. In this case, Eq. (A.26) is modified, i.e.

$$
\begin{aligned}
& H=\frac{e^{\bar{\varphi}}}{L} \int d x w(x), \\
& \dot{\bar{\varphi}}=-\frac{e^{\bar{\varphi}}}{L}\left(x+x_{0}\right) .
\end{aligned}
$$

A particularly simple example of this class of solutions is provided by the profile [2]:

$$
w(x)=\frac{1}{d} \frac{x}{\sqrt{x^{2}+x_{1}^{2}}} .
$$

By integrating the equations of the background with a method similar to the one introduced in the case of constant $w$ solutions, analytical solutions can be found in the case of $V=$ $-V_{0} e^{2 \bar{\varphi}}$. Notice that in the case described by Eq. (A.34) the barotropic index interpolates between $w=-1 / d$ and $w=1 / d$, i.e. for $d=3, w=-1 / 3$ and $w=1 / 3$. The full solution is then:

$$
\begin{aligned}
& \bar{\varphi}=\varphi_{0}-\frac{d}{d-1} \log \left[\left(\frac{x}{x_{1}}\right)^{2}+1\right] \\
& H=\frac{e^{\varphi_{0}}}{d}\left[\left(\frac{x}{x_{1}}\right)^{2}+1\right]^{-\frac{d+1}{2(d-1)}} \\
& \bar{\rho}=\frac{d-1}{d} e^{\varphi_{0}}\left[\left(\frac{x}{x_{1}}\right)^{2}+1\right]^{-\frac{1}{d-1}}
\end{aligned}
$$

\section{B Averages of the energy-momentum tensor}

In this Appendix the averages of the canonical energy-momentum tensor given in Eq. (4.1) will be computed. In the radiation gauge the components of the energy-momentum tensor read

$$
\begin{gathered}
T_{0}^{0}=\frac{1}{2 a^{4}}\left[\left(\mathcal{A}_{i}^{\prime}\right)^{2}+\left(\frac{g^{\prime}}{g}\right)^{2} \mathcal{A}_{i}^{2}+2 \frac{g^{\prime}}{g} \mathcal{A}_{i}^{\prime} \mathcal{A}^{i}+\left(\partial_{i} \mathcal{A}_{j}\right)^{2}-\partial_{i} \mathcal{A}_{j} \partial^{j} \mathcal{A}^{i}\right] \\
T_{i}^{j}=\frac{1}{a^{4}}\left\{\partial_{\eta} \mathcal{A}_{i} \partial_{\eta} \mathcal{A}^{j}+2\left(\frac{g^{\prime}}{g}\right)^{2} \mathcal{A}_{i} \mathcal{A}^{j}+2 \frac{g^{\prime}}{g}\left[\partial_{\eta} \mathcal{A}_{i} \partial_{\eta} \mathcal{A}^{j}\right]\right. \\
-2 \partial_{i} \mathcal{A}_{k} \partial^{j} \mathcal{A}^{k}+2 \partial_{k} \mathcal{A}_{i} \partial^{j} \mathcal{A}^{k}+\frac{\delta_{i}^{j}}{2}\left[-\partial_{\eta} \mathcal{A}_{i} \partial_{\eta} \mathcal{A}^{i}\right.
\end{gathered}
$$




$$
\begin{gathered}
\left.\left.-2 \frac{g^{\prime}}{g} \partial_{\eta} \mathcal{A}_{i} \mathcal{A}^{i}-\left(\frac{g^{\prime}}{g}\right)^{2} \mathcal{A}_{i} \mathcal{A}^{i}+\partial_{i} \mathcal{A}_{k} \partial^{i} \mathcal{A}^{k}-\partial_{i} \mathcal{A}_{k} \partial^{k} \mathcal{A}^{i}\right]\right\} . \\
T_{i}^{0}=\frac{1}{a^{4}}\left[\left(\partial_{\eta} \mathcal{A}^{k}+\frac{g^{\prime}}{g} \mathcal{A}^{k}\right)\left(\partial_{i} \mathcal{A}_{k}-\partial_{k} \mathcal{A}_{i}\right)\right] .
\end{gathered}
$$

Note that in Eqs. (B.1)-(B.3) the spatial indices are flat. Furthermore, in Eqs. (B.2) and (B.3) the derivation with respect to $\eta$ has been indicated with $\partial_{\eta}$ (and not, as usual, with a prime) only in order to avoid confusion with controvariant (flat space) indices.

Recalling now the expression of the field operators given in Eqs. ( 3.4) and ( 3.5), the various expectation values implied by Eqs. (B.1)-(B.3) can be evaluated separately:

$$
\begin{aligned}
& \left\langle\hat{\mathcal{A}}_{i}(\vec{x}, \eta) \hat{\mathcal{A}}_{j}(\vec{y}, \eta)\right\rangle=\int \frac{d^{3} k}{(2 \pi)^{3}} P_{i j}(k)\left|\mathcal{A}_{k}(\eta)\right|^{2} e^{-i \vec{k} \cdot(\vec{x}-\vec{y})}, \\
& \left\langle\Pi_{i}(\vec{x}, \eta) \Pi_{j}(\vec{y}, \eta)\right\rangle=\int \frac{d^{3} k}{(2 \pi)^{3}} P_{i j}(k)\left|\Pi_{k}(\eta)\right|^{2} e^{-i \vec{k} \cdot(\vec{x}-\vec{y})}, \\
& =\int \frac{d^{3} k}{(2 \pi)^{3}} P_{i j}(k)\left[\Pi_{k}(\eta)^{\star} \mathcal{A}_{k}(\eta)+\Pi_{k}(\eta) \mathcal{A}_{k}(\eta)^{\star}\right] e^{-i \vec{k} \cdot(\vec{x}-\vec{y})},
\end{aligned}
$$

where

$$
P_{i j}(k)=\sum_{\alpha} e_{i}^{\alpha} e_{j}^{\alpha}=\delta_{i j}-\frac{k_{i} k_{j}}{k^{2}} .
$$

Using Eqs. (B.4) (for spatially coincident points) into Eqs. (B.1) we obtain

$$
\left\langle T_{0}^{0}(\eta)\right\rangle=\frac{1}{a^{4}} \int \frac{d^{3} k}{(2 \pi)^{3}}\left\{\left|\Pi_{k}\right|^{2}+\left[\left(\frac{g^{\prime}}{g}\right)^{2}+k^{2}\right]\left|\mathcal{A}_{k}\right|^{2}+\frac{g^{\prime}}{g}\left[\Pi_{k}^{*} \mathcal{A}_{k}+\Pi_{k} \mathcal{A}_{k}^{*}\right]\right\},
$$

leading, after angular integration, to Eq. (4.2). The same type of calculation can be performed in the case of the expectation value of Eq. (B.2). The result in this case can be written as

$$
\begin{aligned}
& \left\langle T_{i}^{j}(\eta)\right\rangle=\frac{1}{a^{4}} \int \frac{d^{3} k}{(2 \pi)^{3}}\left\{P_{i}^{j}(k)\left[\left|\Pi_{k}\right|^{2}+\left(\frac{g^{\prime}}{g}\right)^{2}\left|\mathcal{A}_{k}\right|^{2}+\frac{g^{\prime}}{g}\left(\mathcal{A}_{k} \Pi_{k}^{\star}+\mathcal{A}_{k}^{\star} \Pi_{k}\right)\right]\right. \\
& \left.-4 k_{i} k^{j}\left|\mathcal{A}_{k}\right|^{2}+\delta_{i}^{j}\left[\left(k^{2}-\left(\frac{g^{\prime}}{g}\right)^{2}\right)\left|\mathcal{A}_{k}\right|^{2}-\left|\Pi_{k}\right|^{2}-\frac{g^{\prime}}{g}\left(\Pi_{k}^{*} \mathcal{A}_{k}+\Pi_{k} \mathcal{A}_{k}^{*}\right)\right]\right\} .
\end{aligned}
$$

If $f(k, \eta)$ is a generic scalar function of the modulus of the momentum and of the time the following identities hold:

$$
\begin{aligned}
& \int d^{3} k k_{i} k^{j} f(k, \eta)=\frac{4 \pi}{3} \delta_{i}^{j} \int k^{5} f(k, \eta) d \ln k, \\
& \int d^{3} k k^{i} f(k, \eta)=0 .
\end{aligned}
$$

Using Eq. (B.8) into Eq. (B.7) and recalling the explicit expression of the projector $P_{i}^{j}(k)$, we can easily obtain

$$
\left\langle T_{i}^{j}\right\rangle=-\frac{1}{3} \rho_{\mathrm{r}} \delta_{i}^{j} \equiv-p_{\mathrm{r}} \delta_{i}^{j}
$$


as can be argued from the fact that the classical energy-momentum tensor is traceless. Finally, the expectation value $\left\langle T_{i}^{0}\right\rangle$ always vanishes by virtue of Eq. (B.9). 


\section{References}

[1] G. Veneziano, Phys. Lett. B 265, 287 (1991).

[2] M. Gasperini and G. Veneziano, Mod. Phys. Lett. A 8, 3701 (1993)..

[3] M. Gasperini and G. Veneziano, Astropart. Phys. 1, 317 (1993).

[4] K. A. Meissner and G. Veneziano, Mod. Phys. Lett. A 6, 3397 (1991); Phys. Lett. B 267, 33 (1991).

[5] M. Gasperini, J. Maharana and G. Veneziano, Nucl. Phys. B 472, 349 (1996).

[6] M. Gasperini, M. Giovannini and G. Veneziano, Phys. Lett. B 569, 113 (2003)

[7] M. Gasperini, M. Giovannini and G. Veneziano, hep-th/0401112.

[8] M. Gasperini, M. Giovannini and G. Veneziano, Phys. Rev. Lett. 75, 3796 (1995).

[9] G. Veneziano, Strings, Cosmology,... and a particle, contribution to Paschos 1994.

[10] L. Parker, Nature 261, 20 (1976).

[11] L. Parker, Phys. Rev. 183, 1057 (1969).

[12] L. P. Grishchuk, Ann. (N.Y.) Acad. Sci. 302, 439 (1977).

[13] V. N. Lukash and A. A. Starobinsky, Sov. Phys. JETP 39, 742 (1974) [ Zh. Eksp. Teor. Fiz. 66, 1515 (1974)].

[14] L. H. Ford, Phys. Rev. D 35, 2955 (1987).

[15] P. J. E. Peebles and A. Vilenkin, Phys. Rev. D 59, 063505 (1999).

[16] M. Giovannini, Class. Quant. Grav. 16, 2905 (1999); Phys. Rev. D 60, 123511 (1999).

[17] L. H. Ford, Phys. Rev. D 31, 704 (1985).

[18] L. P. Grishchuk, Sov. Phys. JETP 40, 409 (1975) [Zh. Eksp. Teor. Fiz. 67, 825 (1974)].

[19] L. P. Grishchuk, Class. Quant. Grav. 10, 2449 (1993).

[20] C. Lovelace, Phys. Lett. B 135, 75 (1984); E. Fradkin and A. Tseytlin, Nucl. Phys. B 261, 1 (1985); C. Callan at al., Nucl. Phys. B 262, 593 (1985).

[21] A. Erdelyi, W. Magnus, F. Obehettinger, and F. Tricomi, Higher Trascendental Functions (Mc Graw-Hill, New York, 1953). 
[22] W. H. Press, S. A. Teutolsky, W. T. Vetterling, and B. P. Flannery, Numerical Recipes in Fortran 77 (second edition), (Cambridge University Press, Cambridge, 1992 ), p. 731.

[23] R. Grimshaw, Nonlinear Ordinary Differential Equations, (Blackwell Scientific Publications, Oxford, 1990). 